\title{
Effects of a specially designed aerobic dance routine on mild cognitive impairment
}

This article was published in the following Dove Press journal:

Clinical Interventions in Aging

\begin{abstract}
Yi Zhu, ${ }^{1, *} \mathrm{Han} \mathrm{Wu},{ }^{2, *}$ Ming Qi, ${ }^{3}$ Sheng Wang, ${ }^{4}$ Qin Zhang, Li Zhou,' Shiyan Wang, ${ }^{5}$ Wei Wang, ${ }^{6}$ Ting Wu, ${ }^{6}$ Ming Xiao, ${ }^{7}$ Siyu Yang, ${ }^{8}$ Hong Chen, ${ }^{9}$ Ling Zhang, ${ }^{4}$ Kathryn Chu Zhang, ${ }^{10}$ Jinhui Ma, ${ }^{11,12}$ Tong Wang'
\end{abstract}

'Rehabilitation Department, The First Affiliated Hospital of Nanjing Medical University, Nanjing, Jiangsu, China; ${ }^{2}$ Rehabilitation Department, Nanjing Drum Tower Hospital, The Affiliated Hospital of the Medical School at Nanjing University, Nanjing, Jiangsu, China; ${ }^{3}$ Radiology Department, The First Affiliated Hospital of Nanjing Medical University, Nanjing, Jiangsu, China; ${ }^{4}$ Rehabilitation Department, Suzhou Science and Technology Town Hospital, Suzhou, Jiangsu, China; ${ }^{5}$ Rehabilitation Department, Zhejiang Province Hospital, Hangzhou, Zhejiang, China; ${ }^{6}$ Neurological Department, The First Affiliated Hospital of Nanjing Medical University, Nanjing, Jiangsu, China; ${ }^{7}$ Department of Anatomy, Nanjing Medical University, Nanjing, Jiangsu, China; ${ }^{8}$ Neurological Department, The Second Affiliated Hospital of Wan'nan Medical College, Wuhu, Anhui, China; ' $D$ Department of Physical Diagnosis, Nanjing Brain Hospital, Nanjing, Jiangsu, China; ${ }^{10}$ Dalla Lana School of Public Health, University of Toronto, ON, Canada; "School of Epidemiology, Public Health and Preventive Medicine, University of Ottawa, Ottawa, ON, Canada; ${ }^{12}$ Children's Hospital of Eastern Ontario Research Institute, Ottawa, ON, Canada

*These authors contributed equally to this work

\begin{abstract}
Background: Mild cognitive impairment (MCI) is known as a transitional stage or phase between normal aging and dementia. In addition, it is associated with an increased risk of dementia. Research has shown that moderate-intensity exercise is associated with a decreased risk of cognitive impairment. Two recent studies demonstrated that dance interventions are associated with improved cognitive function in the elderly with MCI.
\end{abstract}

Purpose: We evaluated the effect of a moderate-intensity aerobic dance routine on the cognitive function in patients with MCI.

Patients and methods: This is a single-blind randomized controlled trial. Sixty MCI patients were randomized to receive either treatment (aerobic dance routine + usual care) or control (usual care only) for 3 months. All patients received usual care for an additional 3 months thereafter. The aerobic dance routine was a specially designed dance routine which involved cognitive effort for patients to memorize the complex movements. Wechsler memory scale-revised logical memory (WMS-R LM) and event-related evoked potentials (ERPs) P300 latency were used to assess patients' cognitive function at baseline, 3 months, and 6 months.

Results: Twenty-nine patients received exercise therapy and 31 patients received usual care. Patients in the treatment group showed a greater improvement in memory (difference in WMS-R LM changes over 3 months 4.6; 95\% CI 2.2, 7.0; $p<0.001$ ) and processing speed (difference in P300 latency changes over 6 months $-20.0 ; 95 \% \mathrm{CI}=-39.5,-0.4 ; p<0.05)$ compared to control. Conclusion: This dance routine improves cognitive function, especially episodic memory and processing speed, in MCI patients and merits promotion in communities.

Keywords: mild cognitive impairment, dance, cognitive function, memory

\section{Introduction}

The initial diagnostic criteria for mild cognitive impairment (MCI) was published by Petersen et al in 1999, with a focus on memory impairment. ${ }^{1}$ The construct of MCI has evolved over the past decade and broadened to include impairments in other areas of cognitive functions. ${ }^{2,3}$ Nowadays, MCI is categorized into amnestic MCI, where memory loss is the main symptom, and non-amnestic MCI, where memory is not impaired; however, other processing abilities such as organizing, planning, reasoning, learning, or judgment may be affected. ${ }^{4}$ Among people aged $\geq 65$ years, the prevalence of MCI ranges from $16 \%$ to $20 \%$, and the 5-year cumulative incidence ranges from $22.9 \%$ to $30.1 \%{ }^{5,6}$ The amnestic MCI is the most common subtype of MCI, with a prevalence of $10 \%-14 \%$ in the elderly population. ${ }^{5} \mathrm{MCI}$ is considered an intermediate stage between the expected cognitive decline of normal aging and dementia, with a conversion rate of approximately $10 \%$ per year. ${ }^{7}$ People with MCI have an increased risk of developing Alzheimer's disease or dementia, and more than half of them develop clinical dementia during their life. ${ }^{8-11}$ 
To date, no medications have proven effective in treating MCI. ${ }^{12}$ However, some non-pharmacologic interventions, such as a Mediterranean diet, ${ }^{13}$ moderate-intensive exercise, ${ }^{14,15}$ social activities, ${ }^{7,16}$ and cognitive training, ${ }^{17-19}$ have shown promising results through observational research. Recent studies suggest that dance interventions may be more effective in preventing and slowing the progression of MCI because it combines physical, social, and cognitive activities together as well as reduces anxiety and depression. ${ }^{15,20,21}$ Data from recent trials have demonstrated that dance interventions are associated with an improved cognitive function in the elderly with MCI. ${ }^{22,23}$ The dance interventions in these two trials comprise various complex movements that require considerable efforts to learn and employ them properly. However, it is unclear whether it is the effort of learning complex movements or the intensity of the movements that improves cognitive functions to a greater degree. In addition, there is uncertainty with regard to whether cognitive improvement will persist after patients stop dancing. We designed a single-blind randomized controlled trial (RCT) to 1) assess the effectiveness of a specially designed moderate-intensity aerobic dance routine on cognitive functions, compared to the usual care, in patients with amnestic MCI over 3 months, and 2) determine whether the intervention effect persists after the intervention has been discontinued for 3 months.

\section{Patients and methods Study design}

This parallel-group RCT was reviewed and approved by the Ethics Committee of the First Affiliated Hospital of Nanjing Medical University (Jiangsu Provincial People's Hospital). This study was registered on the Chinese Clinical Trial Registry (Registration no.: ChiCTR-INR-15007420). All patients provided written informed consent. The physician who assessed patient outcomes was blinded to the treatment allocation.

\section{Patients}

Patients were recruited through the dementia clinic at the First Affiliated Hospital of Nanjing Medical University or through radio and newspaper recruitment ads. Patients who lived in Nanjing, China, were eligible for participating in this study if they met the following inclusion criteria: 1) age between 50 and 85 years (both inclusive); 2) diagnosed with MCI according to the National Institute on Aging and Alzheimer's Association (NIA-AA) guidelines ${ }^{3}$ and had memory problems for at least 3 months; ${ }^{24} 3$ ) had a Mini-Mental State Examination (MMSE) score $\geq 25$ and a
Montreal Cognitive Assessment (MoCA) score $\leq 26,25,26$ and 4) provided written informed consent.

Patients were excluded from the study if they 1) were diagnosed with vascular dementia based on the National Institute of Neurological Disorders and Stroke and the Association Internationale pour la Recherché et l'Enseignement en Neurosciences (NINDS-AIREN) criteria; ${ }^{27}$ 2) had a Hachinski Ischemic score (HIS) $>4 ;{ }^{24} 3$ ) had disabilities (eg, deafness, blindness, or severe language barriers) which posed significant challenges for cognitive assessments; 4) took any medications in the past 6 months which may cause impaired or improved cognitive performance; 5) were participating in other clinical trials; 6) had unstable hypertension, ischemia myocardial, malignant arrhythmia, or arthralgia of lower limbs; or 7) were considered at high risk, according to the symptom-limited maximum exercise test (see below for details), when carrying out exercises.

The symptom-limited maximal exercise test was conducted before randomization to determine the safety of the dance routine and the maximum heart rate for all patients. In this test, cardiac function and fitness were evaluated using the Modified Bruce Protocol. ${ }^{28}$ Twelve-lead electrocardiography (ECG) and oxygen saturation were monitored during the test. Both the intensity level of physical activity, measured using the Borg Rating of Perceived Exertion scale, ${ }^{29}$ and blood pressure were also recorded. Patients were asked to stop the test and had a 15-minute rest if 1 ) they experienced any discomfort or intolerable fatigue; 2) their ECG showed a horizontal or down-sloping ST depression $\geq 3 \mathrm{~mm}$; 3 ) they had moderate to severe angina pectoris; or 4) their systolic blood pressure dropped $\geq 10 \mathrm{mmHg}$. The maximum heart rate was considered as the heart rate when they stopped the test. If the discomfort, intolerable fatigue, or angina pectoris did not completely alleviate after resting, on taking nitroglycerin, or if the ECG and blood pressure did not return to normal after a 15-minute rest, patients were considered at high risk to undertake the dance routine and, therefore, were excluded from the study.

\section{Randomization}

Patients were randomized on an equal basis to receive either the intervention (perform a dance routine in addition to the usual care) or control (usual care only) based on a computergenerated randomization sequence by an independent statistician. A clinician opened a sequentially numbered, sealed, opaque envelope containing the treatment allocation if the eligibility criteria for enrollment were matched. The unblinded clinician was involved in neither the enrollment nor the outcome assessment. 


\section{Intervention}

All patients received counseling from a physician at baseline, 3 months, and 6 months in promoting a healthy lifestyle to prevent cognitive decline. Moreover, patients in the treatment group were asked to attend a 35-minute dance session 3 times a week for 3 months at the First Affiliated Hospital of Nanjing Medical University. In the first 2 weeks, a dance instructor taught the participants a specially designed dance routine to ensure all participants could perform the dance correctly. Patients danced together with the dance instructor in each session. After 3 months, patients in the treatment group were encouraged to continue practicing the dance routine in their own home.

The target heart rate was set as $60 \%-80 \%$ of the maximum heart rate to ensure safety while producing the desired effect. Patients were asked to wear cardiotachometers (ONrhythm 50, GEONATURE) on their left wrists during each session, and their heart rates were monitored by two physical therapists during the dance class. The dance routine (https://www.youtube.com/watch?v=WuIv1enhtL0), designed by an experienced physical therapist, lasted approximately 35 minutes. The routine included a 5 -minute warm-up, a 25-minute dance with the target heart rate, and a 5-minute cool-down. Patients performed this dance routine in synchronization with a musical phrase of eight rhythmical meters with a general value of $4 / 4$ each. The warm-up session included stepping and breathing, head movement, side bending, and turning exercises. The cooldown session included slow shoulder movement, stepping, and breathing exercises. The dance session included seven sub-sessions performed consecutively: knee bending, heel up, boxing, shoulder movement, kicking, square-stepping, and sculling exercises. Each sub-session comprised six movements (two repetitions of stepping, first and second movements in the sub-session) and was repeated three times. For example, in the kicking sub-session, the first and second movements were left kicking and right kicking, respectively. Concentration and memory were required to perform this dance routine smoothly and correctly.

\section{Outcome measurements}

The outcome measurements were assessed for all patients at baseline, 3 months, and 6 months. Two physicians and one technician who were blinded to the randomization completed all the outcome measurements. All assessments were done at the First Affiliated Hospital of Nanjing Medical University.

\section{Wechsler Memory Scale-Revised logical memory test}

The logical memory (LM) subtest of the Wechsler Memory Scale-Revised (WMS-R) was used to assess patients' memory performance - the primary outcome of this study. In the WMS-R LM subtest, ${ }^{30}$ two short stories (each containing 25 idea units) were read out loud to the patient, who was instructed to recall the details immediately after each story. The WMS-R LM score was calculated as the total number of idea units recalled by the patient for both stories, ranging from 0 to 50. In this study, the WMS-R Fourth Edition of Chinese version (adult battery) was used to assess the logical memory of each patient. ${ }^{31}$

\section{Montreal Cognitive Assessment}

The overall cognitive function was assessed using the Montreal Cognitive Assessment (MoCA) Beijing version (2004), which is a cognitive screening test designed to assist the detection of MCI and Alzheimer's disease and to evaluate aspects of attention, language, verbal memory, visuospatial function, executive function, and orientation. The MoCA score demonstrated high sensitivity and specificity for differentiating individuals with MCI from healthy elderly individuals. ${ }^{32}$ The MoCA score ranged from 0 to 30, with higher scores indicating better cognitive function. A cutoff of 26 (after correction for education) was recommended for the diagnosis of cognitive disorders and 16 for Alzheimer's disease in the United States. ${ }^{33}$

\section{Symbol Digit Modalities Test}

The Symbol Digit Modalities Test (SDMT) - a sensitive instrument for detecting changes in the cognitive function over time and in response to the treatment ${ }^{34}$ - was used to assess sustained and complex attention, informationprocessing speed, and working memory. In this test, patients were required to find the digit corresponding to a specific symbol and to complete a list of 110 symbol-digit pairs. The score was defined as the number of correct answers chosen within 90 seconds.

\section{Trail Making Test}

The Trail Making Test (TMT), which comprises two parts (A and B), was used to evaluate processing speed and executive function. ${ }^{35}$ Both parts of the TMT consisted of 25 circles distributed over a sheet of paper. In Part A (evaluating visual perception abilities, perceptual/motor speed, and speed processing), the circles were numbered 1-25, and patients were asked to draw lines to connect the numbers in ascending order 
(ie, 1-2-3 ... 25). In Part B (evaluating mental flexibility), the circles included both numbers (1-13) and letters (A-L), and patients were asked to connect the circles in an ascending pattern, while alternating between numbers and letters (ie, 1-A-2-B-3-C, etc.). Patients were timed when they connected the "trail".

\section{Forward and backward Digit Span Task}

The forward and backward Digit Span Task (DST; Chinese version) was used to evaluate short-term verbal memory. In the forward DST, a sequence of numbers was read out to the patient, and he/she was then required to repeat these numbers. This process continued until the patient could no longer remember either the full sequence of numbers or in the correct order. In the backward DST, the patient was asked to repeat the sequence in the reverse order. This process was also continued until he/she made an error. The DST was scored by the amount of numbers the patient was able to remember in each test. ${ }^{36,37}$

\section{Functional Activities Questionnaire}

The Functional Activities Questionnaire (FAQ) was used to assess patients' instrumental activities of daily living (IADL). ${ }^{38}$ Patients were asked to rate their IADL on ten categories. Each category was rated as follows: $0=$ normal; $1=$ has difficulty but does by self; $2=$ =requires assistance; and $3=$ dependent. The sum score ranged from 0 to 30 , with higher scores indicating poor performance.

\section{Short Form Health Survey}

The 36-item Short Form Health Survey (SF-36; Chinese version) was used to assess the patient's quality of life across multiple dimensions, including limitations in physical, social, and usual role activities due to physical and emotional problems as well as bodily pain, general mental health, general health perceptions, and vitality. The overall score ranged from 0 to 149 , with lower scores reflecting poor quality of life. ${ }^{39}$

\section{Geriatric Depression Scale}

The 15-item Geriatric Depression Scale (GDS-15) was used to assess the patient's subjective experience of depression in the last week. ${ }^{40}$ Each item in the GDS-15 was scored dichotomously (yes/no). The GDS-15 score ranged from 0 to 15 , with higher scores indicating more depressive symptoms.

\section{Event-related potential}

Event-related potential (ERP), derived from electroencephalography, was used to study the brain neural activity. Auditory
ERP has a defined set of key components from 100 to $600 \mathrm{~ms}$ after the stimulus. The P300 component is the positive ERP that occurs approximately $300 \mathrm{~ms}$ after the onset of a stimulus. ${ }^{41}$ P300 latency (delay between stimulus and response) and amplitude were used as neuropsychological indicators of cognitive impairment because of its objectivity and noninvasiveness. ${ }^{42,43}$ The P300 latency is usually interpreted as the speed of responding to a stimulus - that is, the speed of evaluating the information content of relevant stimulus. Shorter latencies indicate superior cognition performance compared to longer latencies. P300 amplitude reflects stimulus information such that greater attention yields larger P300 waves. Reduced P300 amplitude indicates a broader neurobiological vulnerability. P300 latency has been recognized as an objective and sensitive tool to discriminate patients with MCI from normal aged controls and patients with Alzheimer's disease. Moreover, it can be used as an indicator of cognitive deterioration in longitudinal analyses. ${ }^{44}$ Brain Amp 32 AC system (Brain Products, Gilching, Germany) was used to assess ERP P300 latency and amplitude with a sampling rate set to $500 \mathrm{~Hz}$, impedances kept below $5 \mathrm{k} \Omega$, and scalp positions placed according to the international 10-20 system. The P300 latency and amplitude were measured using Brain Vision Analyzer 2.0 software (Brain Products, Munich, Germany).

\section{Adverse events}

Patients were questioned about adverse events at each study visit. Research staff recorded all directly observed adverse events and those reported by patients.

\section{Sample size calculation}

Changes in memory (measured using the WMS-R LM) at 3 months was considered as the primary outcome for sample size calculation. To detect a moderate effect size of $0.75 \mathrm{SD}$, a minimum sample size of 56 (28 per group) was required to achieve $80 \%$ statistical power at the significance level of 0.05 (two-sided).

\section{Statistical analysis}

Analyses were conducted using SAS, version 9.3 (SAS Institute Inc., Cary, NC, USA). Categorical variables were summarized using frequency and percentage. Continuous variables were summarized using mean and SD or median and interquartile range (IQR), as appropriate. For the primary analysis, linear mixed-effects modeling for repeated measures over time was used, with the WMS-R LM score as the dependent variable and time, group, and time-by-group interaction as independent variables. Changes within each 
group over time and the intergroup contrast in the WMS-R LM score, and the corresponding 95\% CIs, were reported. The same analysis was conducted for MoCA, forward and backward DST, TMT Part A/B, SDMT, SF-36 score, FAQ, GDS-15, and P300 latency and amplitude.

\section{Results}

We used the CONSORT statement to facilitate complete and transparent reporting of this trial. ${ }^{45}$ Recruitment started in June 2014 and closed in January 2015. The final follow-up visit was in July 2015. We screened 112 adults who experienced memory loss or other cognitive impairments and showed interest in this study. Fifty-two were excluded due to either not meeting the inclusion criteria $(n=51)$ or meeting the inclusion criteria but losing interest in participating in the study $(n=1)$. Sixty patients were randomized to receive either the intervention $(n=29)$ or control $(n=31)$. The flow of patients through the trial and the details of dropouts are shown in Figure 1.

From baseline to 3 months, a total of 36 dance sessions were offered to the patients in the intervention group. The median number of sessions that patients attended was $36(\mathrm{IQR}=34,36)$. Every patient attended at least 32 dance sessions. No adverse events were reported or observed over the 6 months of the study period.

\section{Patient characteristics at baseline}

Of the 60 patients (mean age $\pm \mathrm{SD}=69.6 \pm 7.0$ years), $36(60 \%)$ were female and $53(88 \%)$ had a high school or higher

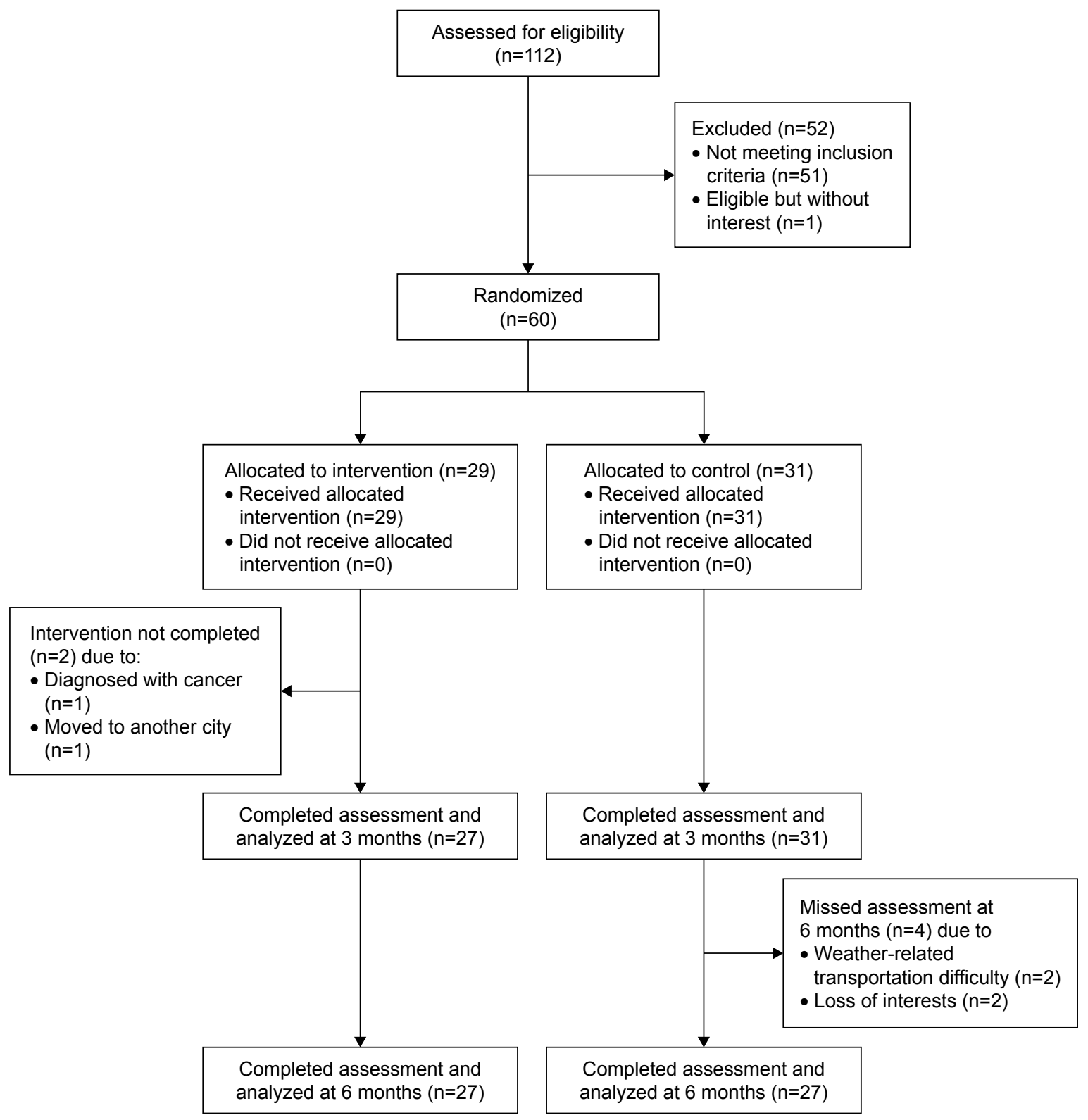

Figure I Patient disposition during the trial. 
education level. The characteristics of patients in the intervention and control groups were similar at baseline (Table 1). Moreover, the cognitive function, quality of life, and physical activity parameters were comparable between patients in different groups (Table 2).

\section{Within-group comparison of patient outcomes}

Patients in the intervention group improved significantly at 3 months in memory (mean increase in WMSR-LR 3.4; 95\% CI 1.6, 5.1; $p<0.05$ ), processing speed (mean changes in P300 latency $-17.3 \mathrm{~ms} ; 95 \% \mathrm{CI}-31.1,-3.5 ; p<0.05)$, and cognitive function (mean increase in MoCA $1.6 ; 95 \% \mathrm{CI}$ $0.8,2.3 ; p<0.001)$. These improvements were still observable at 5 months. Moreover, their quality of life at 6 months improved significantly compared to that at baseline (mean increase in SF-36 score 6.1, 95\% CI 1.1, 11.2, $p<0.05$ ) (Table 2).

Patients in the control group had a significant improvement at 3 months in the cognitive function (mean increase in MoCA $0.7 ; 95 \%$ CI $0.0,1.4 ; p<0.05$ ) and depression symptoms (mean GDS-15 score change $-3.4,95 \%$ CI -5.2, -1.5; $p<0.01)$. A similar improvement was observed at 6 months as well. However, patients' memory (mean change in forward and backward DST $-1.2,95 \% \mathrm{CI}-2.2,-0.2 ; p<0.05)$ and processing speed (mean increase in P300 latency 15.6, 95\% CI 1.7, 29.4; $p<0.05$ ) worsened at 6 months (Table 2).

\section{Between-group comparison of patient outcomes}

Compared to patients in the control group, patients in the intervention group showed a greater improvement in episodic memory at 3 months (difference between the changes in WMSR-LM 4.6, 95\% CI 2.2, 7.0; $p<0.001$ ) and in processing speed at 6 months (difference between the changes in P300 latency $-20.0,95 \% \mathrm{CI}-39.5,-0.4 ; p<0.05$ ) (Table 2).

\section{Discussion}

This study demonstrates that patients who participated in this 3-month, moderate-intensity, aerobic dance routine had a greater decrease in the duration of stimulus evaluation (ie, a greater increase in the processing speed) and more improvement in episodic memory compared to those under usual care only. Our dance routine consists of a sequence of movements assembled in a specific order. Learning how to combine dance steps requires practice (repetition) and cognitive efforts including concentration, consciousness, and, particularly, episodic memory. This may be the cause of improved processing speed and episodic memory among those in the dance group compared to those in the control group. Doi et al reported that dance was associated with a significant improvement in story memory and global cognition among patients with amnestic MCI. ${ }^{22}$ Furthermore, our results confirm these findings that dancing has a beneficial effect on the cognitive function in MCI patients. ${ }^{22,23}$ Compared

Table I Patient characteristics at baseline

\begin{tabular}{|c|c|c|c|}
\hline Characteristics & $\begin{array}{l}\text { Treatment group } \\
(\mathrm{n}=29)\end{array}$ & $\begin{array}{l}\text { Control group } \\
(n=31)\end{array}$ & $p$-value \\
\hline Age (years), mean (SD) & $70.3(6.7)$ & $69.0(7.3)$ & 0.47 \\
\hline Female, n (\%) & $15(51.7 \%)$ & $21(67.7 \%)$ & 0.29 \\
\hline Height $(\mathrm{cm})$, mean $(\mathrm{SD})$ & $154.8(9.6)$ & I57.I (8.9) & 0.42 \\
\hline Weight (kg), mean (SD) & $58.1(8.8)$ & $58.3(10.5)$ & 0.30 \\
\hline Six-minute walking test $(\mathrm{m})$, mean $(\mathrm{SD})$ & $5.7(6.8)$ & $5.4(6.4)$ & 0.17 \\
\hline $\begin{array}{l}\text { Timed I0-m walk test (with self- } \\
\text { selected velocity) }(\mathrm{m} / \mathrm{s}) \text {, mean }(\mathrm{SD})\end{array}$ & $7.0(1.1)$ & $7.7(1.2)$ & 0.08 \\
\hline $\begin{array}{l}\text { Timed I0-m walk test (in fast- } \\
\text { velocity) }(\mathrm{m} / \mathrm{s}), \text { mean }(\mathrm{SD})\end{array}$ & $5.5(1.0)$ & $5.5(0.8)$ & 0.98 \\
\hline High blood pressure*, n (\%) & $8(47.1 \%)$ & $18(72.0 \%)$ & 0.12 \\
\hline Hachinski Ischemia Scale & & & 0.27 \\
\hline 0 & $12(4 \mid .4 \%)$ & $7(22.6 \%)$ & \\
\hline 1 & II (37.9\%) & $13(41.9 \%)$ & \\
\hline$\geq 2$ & $6(20.7 \%)$ & II (35.5\%) & \\
\hline Education level, n (\%) & & & 0.42 \\
\hline High school or less & $4(13.8 \%)$ & $3(9.7 \%)$ & \\
\hline College/university & II (37.9\%) & $17(54.8 \%)$ & \\
\hline Postgraduate & $14(48.3 \%)$ & II (35.5\%) & \\
\hline
\end{tabular}

Note: *High blood pressure is defined as systolic blood pressure $>140 \mathrm{mmHg}$ or diastolic blood pressure $>90 \mathrm{mmHg}$. 
Table 2 Comparison of clinical parameters between two study groups

\begin{tabular}{|c|c|c|c|c|c|c|c|}
\hline \multirow[t]{2}{*}{$\begin{array}{l}\text { Outcome } \\
\text { by group }\end{array}$} & \multicolumn{3}{|c|}{$\begin{array}{l}\text { Summary at different time point }{ }^{\mathrm{a}} \\
\text { mean (SD) }\end{array}$} & \multicolumn{2}{|c|}{$\begin{array}{l}\text { From baseline to } 3 \text { months } \\
\text { mean }(95 \% \mathrm{Cl})\end{array}$} & \multicolumn{2}{|c|}{$\begin{array}{l}\text { From baseline to } 6 \text { months } \\
\text { mean }(95 \% \mathrm{Cl})\end{array}$} \\
\hline & Baseline & 3 months & 6 months & $\begin{array}{l}\text { Within-group } \\
\text { change }^{\&}\end{array}$ & $\begin{array}{l}\text { Between-group } \\
\text { difference in change } e^{\ddagger}\end{array}$ & $\begin{array}{l}\text { Within-group } \\
\text { change }^{\&}\end{array}$ & $\begin{array}{l}\text { Between-group } \\
\text { difference in change } \mathrm{e}^{\ddagger}\end{array}$ \\
\hline \multicolumn{8}{|c|}{ Wechsler Memory Scale-Revised, Logical Memory } \\
\hline Exercise & $14.3(5.4)$ & $17.5(3.8)$ & $16.7(6.3)$ & $3.4(I .6,5.1)^{*}$ & $4.6(2.2,7.0)^{* *}$ & $2.8(0.9,4.6)^{*}$ & $2.8(0.2,5.4)$ \\
\hline Control & $15.5(5.2)$ & I $4.3(4.3)$ & $15.5(5.4)$ & $-1.2(-2.9,0.4)$ & & $0.0(-1.8, I .8)$ & \\
\hline \multicolumn{8}{|c|}{ Montreal Cognitive Assessment } \\
\hline Exercise & $23.2(1.9)$ & $24.7(2.2)$ & $25.0(2.4)$ & $1.6(0.8,2.3)^{* *}$ & $0.8(-0.1,1.8)$ & $1.9(1.0,2.8)^{* *}$ & $0.2(-1.0,1.4)$ \\
\hline Control & $22.9(2.1)$ & $23.6(1.8)$ & $24.7(2.4)$ & $0.7(0.0,1.4)^{*}$ & & $1.7(0.8,2.6)^{* *}$ & \\
\hline \multicolumn{8}{|c|}{ Digit Span, forward and backward } \\
\hline Exercise & $16.8(2.7)$ & $16.9(2.3)$ & $16.8(2.2)$ & $0.1(-I .1,1.3)$ & $0.2(-1.4,1.9)$ & $0.0(-1.0,1.0)$ & I.2(-0.2, 2.6) \\
\hline Control & $17.2(2.9)$ & $17.0(2.9)$ & $15.9(3.0)$ & $-0.2(-1.3,0.9)$ & & $-1.2(-2.2,-0.2)^{*}$ & \\
\hline \multicolumn{8}{|c|}{ Trail Making Test, Part A } \\
\hline Exercise & $74(29)$ & $66(25)$ & $60(20)$ & $-8.7(-17.2,0.2)$ & $-7.4(-19.0,4.3)$ & $-15(-24,-6)^{*}$ & $-10(-23,2)$ \\
\hline Control & $70(23)$ & $69(20)$ & $66(22)$ & $-1.3(-9.4,6.7)$ & & $-4.9(-13.6,3.8)$ & \\
\hline \multicolumn{8}{|c|}{ Trail Making Test, Part B } \\
\hline Exercise & $200(73)$ & $158(49)$ & $|7|(9 \mid)$ & $-42(-62,23)$ & $-32(-59,-4)^{*}$ & $-30(-52,7)$ & $-20(-52,11)$ \\
\hline Control & $187(67)$ & $177(48)$ & $179(69)$ & $-11(-30,8)$ & & $-9(-32,13)$ & \\
\hline \multicolumn{8}{|c|}{ Symbol Digit Modalities Test } \\
\hline Exercise & $32(9)$ & $36(9)$ & $37(9)$ & $3.6(0.8,6.5)^{*}$ & $3.4(-0.6,7.3)$ & $5.2(2.3,8.0) *$ & I.7 $(-2.3,5.7)$ \\
\hline Control & $33(\mathrm{II})$ & $33(11)$ & $35(13)$ & $0.3(-2.4,2.9)$ & & $3.5(0.6,6.3)^{*}$ & \\
\hline \multicolumn{8}{|c|}{ Short Form Health Survey-36 } \\
\hline Exercise & $108(17)$ & $114(17.7)$ & $115(18)$ & $5.2(0.1,10.4)$ & $2.4(-4.6,9.4)$ & $6.1(1.1,11.2)^{*}$ & $5.6(-1.5,12.7)$ \\
\hline Control & $109(15)$ & $112(14.7)$ & III (I7) & $2.8(-2.0,7.7)$ & & $0.5(-4.5,5.5)$ & \\
\hline \multicolumn{8}{|c|}{ Functional Activities Questionnaire } \\
\hline Exercise & $1.4(2.2)$ & $0.7(1.5)$ & $0.7(1.7)$ & $-0.7(-1.7,0.2)$ & $0.1(-1.2,1.3)$ & $-0.7(-1.7,0.3)$ & $0.6(-0.8,2.0)$ \\
\hline Control & $2.0(2.4)$ & $1.2(1.9)$ & $0.7(1.2)$ & $-0.8(-1.7,0.1)$ & & $-1.3(-2.3,0.3)$ & \\
\hline \multicolumn{8}{|c|}{ Geriatric Depression Scale-15 } \\
\hline Exercise & $12.3(7.2)$ & $10.4(6.0)$ & $10.2(7.0)$ & $-1.9(-3.9,0.0)$ & I.4 $(-1.3,4.1)$ & $-2.1(-4.2,0.1)$ & $1.2(-1.9,4.2)$ \\
\hline Control & $14.5(6.9)$ & $11.2(6.0)$ & $1 \mathrm{I} .2(6.7)$ & $-3.4(-5.2,-1.5)^{* *}$ & & $-3.2(-5.4,-1.1)^{*}$ & \\
\hline \multicolumn{8}{|c|}{ P300 Latency (ms) } \\
\hline Exercise & $440(34)$ & $424(40)$ & $435(26)$ & $-17(-3 \mid,-4)^{*}$ & $-7(-26,12)$ & $-4.4(-18.2,9.4)$ & $-20(-40,-0.4)^{*}$ \\
\hline Control & $431(38)$ & $421(38)$ & $445(23)$ & $-10(-24,3)$ & & $15.6(1.7,29.4)^{*}$ & \\
\hline \multicolumn{8}{|c|}{ P300 Amplitude $(\mu v)$} \\
\hline Exercise & $7.9(5.8)$ & $7.3(3.9)$ & $8.9(4.9)$ & $-0.4(-2.7,1.8)$ & $-1.2(-4.3,1.9)$ & I.2 (-I.I, 3.5) & $0.1(-3.2,3.4)$ \\
\hline Control & $8.8(5.7)$ & $9.4(6.9)$ & $9.6(6.9)$ & $0.8(-1.4,2.9)$ & & I.I $(-I .3,3.5)$ & \\
\hline
\end{tabular}

Notes: ${ }^{*} 0.00 \mathrm{I} \leq p<0.05 ;{ }^{* *} p<0.00 \mathrm{I} ;{ }^{\circledR}$ Within-group change is calculated as the outcome measure at follow-up minus the measure at baseline; ${ }^{\ddagger}$ Between-group difference in change is calculated as the change over the follow-up period in the intervention group minus the change in the control group.

to the previous trials, which emphasized the variability and complexity of the dance movements, our trial focused on the intensity of the dance movements and ensured patients achieved their target heart rates at each training session. As the prolonged P300 latency in MCI patients implies the increased information-processing time,${ }^{46-48}$ our findings indicated aerobic dance could improve patients' processing speed. Moreover, we followed up patients for another 3 months after they discontinued the dance intervention and found that the memory improvement that was previously achieved was attenuated. The processing speed of patients in the dance group (measured using the P300 measure) returned to their baseline levels. This may be due to decreased intensity when practicing the dance at home between 3 and 6 months. In contrast, the processing speed of patients in the control group showed a significant decrease by 6 months. These findings highlight two major findings: first, it is essential to perform the moderate-intensity dance routine regularly in order to maintain its beneficial effects, and, second, dance sessions offered at local community centers may be helpful in facilitating attendance in the older population. Further investigation is needed to understand the mechanism by which the dance intervention benefits the MCI patients - that is, whether the changes of rhythm and the spatial awareness in the dance 
movements activate place cells and grid cells, or the aerobic exercise delays the degeneration of nerve cells. ${ }^{49-51}$

Moderate-intensity exercise has been shown to have a positive effect on the cognitive function in patients with MCI and delay the onset of $\mathrm{AD} ;{ }^{52,53}$ however, it tends to be disregarded by many people, especially those with MCI and a risk of AD. In addition, the effect of exercise on the cognitive function depends on how often the exercise is performed. Exercising regularly, as opposed to exercising occasionally, is essential in order to experience an improvement in the cognitive function. However, it can be challenging for elderly people to exercise on a regular basis due to lack of motivation and enjoyment. A previous study reported an adherence rate of $79.2 \%$ to a moderate-intensity exercise program in elderly people with MCI. ${ }^{15}$ The dance routine in our study, performed with peers in synchronization with rhythmic music, can provide an enhanced environment and, therefore, is more attractive and enjoyable for elderly people. In addition, this dance routine can be easily organized at community centers; thus, people can attend the dance sessions conveniently at low or no cost. We believe this dance routine will bring considerable economic and social benefits when applied in communities.

We acknowledge that there are some limitations of this study. First, we could not demonstrate that the dance routine was significantly superior to usual care in improving patients' overall cognitive function due to the lack of statistical power. However, we were able to show that overall cognitive function was improved for patients in both groups, and the improvement was substantially more for patients in the intervention group. Second, we focused on patients with amnestic MCI only, and our findings may not apply to those with non-amnestic MCI.

A recent review by Karkou and Meekums suggests that trials of high methodological quality, large sample sizes, and clarity in the way the intervention is put together and delivered are needed to assess whether dance interventions are an effective intervention for dementia. ${ }^{54}$ The present trial shows that this specially designed aerobic dance routine improves the cognitive function, especially episodic memory and processing speed, in patients with MCI. Our results suggest that dance movement therapy may be a promising intervention for the treatment of dementia in the early stages of cognitive impairment.

\section{Acknowledgments}

This study was supported by the Science and Technology Department of Jiangsu Province (Project Number: 2013DB13). The authors thank Chenjin Yan and Lixia Zhang from the Clinical Laboratory Department of the laboratory of the First Affiliated Hospital of Nanjing Medical University for completing the laboratory tests for all the participants.

\section{Disclosure}

The authors report no conflicts of interest in this work.

\section{References}

1. Petersen RC, Smith GE, Waring SC, Ivnik RJ, Tangalos EG, Kokmen E. Mild cognitive impairment: clinical characterization and outcome. Arch Neurol. 1999;56(3):303-308.

2. Winblad B, Palmer K, Kivipelto M, et al. Mild cognitive impairment - beyond controversies, towards a consensus: report of the International Working Group on Mild Cognitive Impairment. J Intern Med. 2004;256(3):240-246.

3. Petersen RC, Caracciolo B, Brayne C, Gauthier S, Jelic V, Fratiglioni L. Milde cognitive impairment: a concept in evolution. J Intern Med. 2014;275(3):214-228.

4. Albert MS, DeKosky ST, Dickson D, et al. The diagnosis of mild cognitive impairment due to Alzheimer's disease: recommendations from the National Institute on Aging-Alzheimer's Association workgroups on diagnostic guidelines for Alzheimer's disease. Alzheimers Dement. 2011;7(3):270-279.

5. Roberts R, Knopman DS. Classification and epidemiology of MCI. Clin Geriatr Med. 2013;29(4):753-772.

6. Ward A, Arrighi HM, Michels S, Cedarbaum JM. Mild cognitive impairment: disparity of incidence and prevalence estimates. Alzheimers Dement. 2012;8(1):14-21.

7. Eshkoor SA, Hamid TA, Mun CY, Ng CK. Mild cognitive impairment and its management in older people. Clin Interv Aging. 2015;10(10): 687-693.

8. Lopez OL, Jagust WJ, DeKosky ST, et al. Prevalence and classification of mild cognitive impairment in the Cardiovascular Health Study Cognition Study: part 1. Arch Neurol. 2003;60(10):1385-1389.

9. Purser JL, Fillenbaum GG, Pieper CF, Walloce RB. Mild cognitive impairment and 10-year trajectories of disability in the Iowa established populations for epidemiologic studies of the elderly cohort. J Am Geriatr Soc. 2005;53(11):1966-1972.

10. Pérès K, Chrysostome V, Fabrigoule C, Orgogozo JM, Dartigues JF, Barberger-Gateau P. Restriction in complex activities of daily living in MCI: impact on outcome. Neurology. 2006;67(3):461-466.

11. Busse A, Angermeyer MC, Riedel-Heller SG. Progression of mild cognitive impairment to dementia: a challenge to current thinking. Br J Psychiatry. 2006;189(11):399-404.

12. Cooper C, Li R, Lyketsos C, Livingston G. Treatment for mild cognitive impairment: systematic review. Br J Psychiatry. 2013;203(3): 255-264.

13. Roberts RO, Geda YE, Cerhan JR, et al. Vegetables, unsaturated fats, moderate alcohol intake, and mild cognitive impairment. Dement Geriatr Cogn Disord. 2010;29(5):413-423.

14. Geda YE, Roberts RO, Knopman DS, et al. Physical exercise, aging, and mild cognitive impairment: a population-based study. Arch Neurol. 2010;67(1):80-86.

15. Suzuki T, Shimada H, Makizako H, et al. Effects of multicomponent exercise on cognitive function in older adults with amnestic mild cognitive impairment: a randomized controlled trial. BMC Neurol. 2012;12(10): $128-135$.

16. Odawara T. Cautious notification and continual monitoring of patients with mild cognitive impairment. Psychogeriatrics. 2012;12(2): 131-132.

17. Hampstead BM, Stringer AY, Stilla RF, Giddens M, Sathian K. Mnemonic strategy training partially restores hippocampal activity in patients with mild cognitive impairment. Hippocampus. 2012;22(8): $1652-1658$. 
18. Greenaway MC, Duncan NL, Smith GE. The memory support system for mild cognitive impairment: randomized trial of a cognitive rehabilitation intervention. Int J Geriatr Psychiatry. 2013;28(4): 402-409.

19. Moro V, Condoleo MT, Valbusa V, Broggio E, Moretto G, Gambina G. Cognitive stimulation of executive functions in mild cognitive impairment: specific efficacy and impact in memory. Am J Alzheimers Dis Other Demen. 2015;30(2):153-164.

20. Dhami P, Moreno S, DeSouza JF. New framework for rehabilitation fusion of cognitive and physical rehabilitation: the hope for dancing. Front Psychol. 2015;5(1):1478.

21. Adam D, Ramli A, Shahar S. Effectiveness of a combined dance and relaxation intervention on reducing anxiety and depression and improving quality of life among the cognitively impaired elderly. Sultan Qaboos Univ Med J. 2016;16(1):e47-e53.

22. Doi T, Verghese J, Makizako H, et al. Effects of cognitive leisure activity on cognition in mild cognitive impairment: results of a randomized controlled trial. J Am Med Dir Assoc. 2017;8(8):686-691.

23. Lazarou I, Parastatidis T, Tsolaki A, et al. International ballroom dancing against neurodegeneration: a randomized controlled trial in Greek community-dwelling elders with mild cognitive impairment. Am J Alzheimers Dis Other Demen. 2017;32(8):489-499.

24. Zhang H, Zhao L, Yang S, et al. Clinical observation on effect of scalp electroacupuncture for mild cognitive impairment. J Tradit Chin Med. 2013;33(1):46-50.

25. Folstein MF, Folstein SE, McHugh PR. "Mini-mental state". A practical method for grading the cognitive state of patients for the clinician. 1975;12(3):189-198

26. Yu J, Li J, Huang X. The Beijing version of the Montreal Cognitive Assessment as a brief screening tool for mild cognitive impairment: a community-based study. BMC Psychiatry. 2012;12(9):156.

27. Ryu HG, Youn SW, Kwon OD. Lack of association between apolipoprotein E polymorphism with age at onset of subcortical vascular dementia. Dement Geriatr Cogn Dis Extra. 2012;2(1):1-9.

28. Okin PM, Ameisen O, Kligfield P. A modified treadmill exercise protocol for computer-assisted analysis of the ST segment/heart rate slope: methods and reproducibility. J Electrocardiol. 1986;19(4):311-318.

29. Hamilton AL, Killian KJ, Summers E, Jones NL. Symptom intensity and subjective limitation to exercise in patients with cardiorespiratory disorders. Chest. 1996;110(5):1255-1263.

30. Sullivan K. Estimates of interrater reliability for the logical memory subtest of the Wechsler Memory Scale-revised. J Clin Exp Neuropsychol. 1996;18(5):707-712.

31. Wang J, Zou YZ, Cui JF, et al. Revision of the Wechsler memory scalefourth edition of Chinese version (adult battery). Chin Ment Health J. 2015;29(1):53-59.

32. Luis CA, Keegan AP, Mullan M. Cross validation of the Montreal Cognitive Assessment in community dwelling older adults residing in the Southeastern US. Int Geriatr Psychiatry. 2009;24(2):197-201.

33. Nasreddine ZS, Phillips NA, Bédirian V, et al. The Montreal Cognitive Assessment, MoCA: a brief screening tool for mild cognitive impairment. J Am Geriatr Soc. 2005;53(4):695-699.

34. Cherbuin N, Sachdev P, Anstey KJ. Neuropsychological predictors of transition from healthy cognitive aging to mild cognitive impairment: the PATH through life study. Am J Geriatr Psychiatry. 2010;18(8): 723-733.

35. Perrochon A, Kemoun G. The Walking Trail-Making Test is an early detection tool for mild cognitive impairment. Clin Interv Aging. 2014; 9(1):111-119.
36. Laures-Gore J, Marshall RS, Verner E. Performance of individuals with left-hemisphere stroke and aphasia and individuals with right brain damage on forward and backward digit span tasks. Aphasiology. 2011;25(1):43-56.

37. Hester RL, Kinsella GJ, Ong B. Effect of age on forward and backward span tasks. J Int Neuropsychol Soc. 2004;10(4):475-481.

38. Hsiao JJ, Lu PH, Grill JD, Teng E. Longitudinal declines in instrumental activities of daily living in stable and progressive mild cognitive impairment. Dement Geriatr Cogn Disord. 2015;39(1-2) $12-24$.

39. Lam CL, Gandek B, Ren XS, Chan MS. Tests of scaling assumptions and construct validity of the Chinese (HK) version of the SF-36 Health Survey. J Clin Epidemiol. 1998;51(11):1139-1147.

40. de Paula JJ, Bicalho MA, Avila RT, et al. A reanalysis of cognitivefunctional performance in older adults: investigating the interaction between normal aging, mild cognitive impairment, mild Alzheimer's disease dementia, and depression. Front Psychol. 2015;6(1):2061.

41. Polich J. Updating P300: an integrative theory of P3a and P3b. Clin Neurophysiol. 2007;118(10):2128-2148.

42. Rugg MD, Coles MGH. Event-related brain potentials: an introduction. In: Rugg MD, Coles MGH, editors. Electrophysiology of Mind: EventRelated Brain Potentials and Cognition. New York: Oxford University Press; 1995.

43. Polich J. Meta-analysis of P300 normative aging studies. Psychophysiol. 1996;33(4):334-353.

44. Jiang S, Qu C, Wang F, et al. Using event-related potential P300 as an electrophysiological marker for differential diagnosis and to predict the progression of mild cognitive impairment: a meta-analysis. Neuro Sci. 2015;36(7):1105-1112.

45. Schulz KF, Altman DG, Moher D; CONSORT Group. CONSORT 2010 statement: updated guidelines for reporting parallel group randomised trials. PLoS Med. 2010;7(3):e1000251.

46. Howe AS, Bani-Fatemi A, De Luca V. The clinical utility of the auditory P300 latency subcomponent event-related potential in preclinical diagnosis of patients with mild cognitive impairment and Alzheimer's disease. Brain Cogn. 2014;86:64-74.

47. Golob EJ, Johnson JK, Starr A. Auditory event-related potentials during target detection are abnormal in mild cognitive impairment. Clin Neurophysiol. 2002;113(1):151-161.

48. Parra MA, Ascencio LL, Urquina HF, Manes F, Ibáñez AM. P300 and neuropsychological assessment in mild cognitive impairment and Alzheimer dementia. Front Neurol. 2012;3:172.

49. Doeller CF, Barry C, Burgess N. Evidence for grid cells in a human memory network. Nature. 2010;463(7281):657-661.

50. Hafting T, Fyhn M, Molden S, Moser MB, Moser EI. Microstructure of a spatial map in the entorhinal cortex. Nature. 2005;436(7052): 801-806.

51. Colcombe SJ, Erickson KI, Raz N, Webb AG, Cohen NJ, McAuley E, Kramer AF. Aerobic fitness reduces brain tissue loss in aging humans. J Gerontol A Biol Sci Med Sci. 2003;58(2):176-180.

52. Zheng G, Xia R, Zhou W, Tao J, Chen L. Aerobic exercise ameliorates cognitive function in older adults with mild cognitive impairment: a systematic review and meta-analysis of randomised controlled trials. Br J Sports Med. 2016 Epub Apr 19.

53. Groot C, Hooghiemstra AM, Raijmakers PG, et al. The effect of physical activity on cognitive function in patients with dementia: a meta-analysis of randomized control trials. Ageing Res Rev. 2016;25:13-23.

54. Karkou V, Meekums B. Dance movement therapy for demential. Cochrane Database Syst Rev. 2017;2:CD011022. 


\section{Publish your work in this journal}

Clinical Interventions in Aging is an international, peer-reviewed journal focusing on evidence-based reports on the value or lack thereof of treatments intended to prevent or delay the onset of maladaptive correlates of aging in human beings. This journal is indexed on PubMed Central, MedLine,

CAS, Scopus and the Elsevier Bibliographic databases. The manuscript management system is completely online and includes a very quick and fair peer-review system, which is all easy to use. Visit http://www.dovepress. com/testimonials.php to read real quotes from published authors. 